\title{
The Association Between Hyperhomocysteinemia and Thyroid Nodule Prevalence in an Adult Population
}

\author{
Qingrong Pan, MD, ${ }^{1}$ Ying Wang, MD, ${ }^{2}$ and Guang Wang, $\mathrm{MD}^{1}$
}

\begin{abstract}
Background: Thyroid nodule is the most common disorder of thyroid. Metabolic syndrome was regarded as an important factor for the prevalence of thyroid nodule. Homocysteine has been shown to be related to metabolic syndrome, inflammation, and several common cancers. In this study, we aimed to investigate the relationship between serum homocysteine and the prevalence of thyroid nodule.

Materials and Methods: This was a cross-sectional study that included 2040 adults in a health checkup population in Beijing Chao-yang hospital. Thyroid ultrasound data, together with anthropometric characteristics, metabolic parameters, and serum homocysteine, were recorded respectively.

Results: Hyperhomocysteinemia (defined as serum homocysteine $\geq 15 \mu \mathrm{mol} / \mathrm{L}$ ) was detected in 452 participants $(21.91 \%)$. Thyroid nodule prevalence was significantly higher in hyperhomocysteinemia participants than in normal homocysteine participants $(52.57 \%$ vs. $45.16 \%, P=0.006)$. Logistic regression analysis revealed that age [odds ratio (OR) 1.054; $P<0.001$ ], female gender (OR 2.242; $P<0.001$ ), body mass index (OR 1.050; $P<0.001)$, and serum homocysteine level (OR 1.022; $P=0.001)$ were the independent risk factors for thyroid nodule.

Conclusions: Subjects with hyperhomocysteinemia have significantly higher thyroid nodule prevalence. Homocysteine is an independent risk factor for thyroid nodule. It implies that individuals with hyperhomocysteinemia have higher susceptibility to thyroid nodule.
\end{abstract}

Keywords: thyroid nodule, homocysteine, hyperhomocysteinemia, metabolic syndrome, gender

\section{Background}

A N INCREASING PREVALENCE of thyroid nodule was observed with the popularity of health checkup and the increased use of thyroid ultrasound examination. ${ }^{1}$ It has been reported that the prevalence of thyroid nodule, based on ultrasonography, is from $18.6 \%$ to $49 \%$ in China. ${ }^{2-4}$ Although most of thyroid nodule are benign, $5 \%-10 \%$ of them are malignant. ${ }^{5,6}$ The risk factors for thyroid nodule have received much attention. Advanced age, female gender, and obesity have been reported to increase the susceptibility for thyroid nodule. ${ }^{3,7}$ In addition, it has also been suggested that the metabolic syndrome and its components are associated with thyroid nodule prevalence. .,9 $^{8}$

Homocysteine is a nonprotein-forming amino acid, an important component in one carbon metabolism. In healthy population, plasma homocysteine level is $<15 \mu \mathrm{mol} / \mathrm{L}$. Hyperhomocysteinemia is characterized as an elevated level of toxic homocysteine in the plasma. Previous studies have revealed that hyperhomocysteinemia is an independent risk factor of coronary heart disease. ${ }^{10}$ In addition, hyperhomocysteinemia was also reported to be associated with metabolic syndrome, obesity, insulin resistance, and inflammation. ${ }^{11-13}$ Recent advances have demonstrated that there is a close link between hyperhomocysteinemia and several types of common cancers. ${ }^{14-17}$ However, to our best knowledge, no report on the relationship between homocysteine and thyroid nodule prevalence has been published.

This study was designed to investigate the correlation between serum homocysteine level, hyperhomocysteinemia, and thyroid nodule prevalence in a health checkup population of Beijing.

\footnotetext{
${ }^{1}$ Department of Endocrinology, Beijing Chao-Yang Hospital, Capital Medical University, Beijing, China.

${ }^{2}$ Physical Examination Center, Beijing Chao-Yang Hospital, Capital Medical University, Beijing, China.
} 


\section{Materials and Methods}

\section{Study population}

We retrospectively analyzed the records of individuals older than 18 years, who had undergone thyroid ultrasonography as part of a routine health checkup in Beijing Chao-yang Hospital, Capital Medical University, from March to October 2017. The participants with thyroid dysfunction or under treatment with thyroxine or anti-thyroid were excluded. A total of 2040 individuals were included in the study. The study received approval from the Ethics Committee of Beijing Chao-yang Hospital and implemented in accordance with the 1964 Helsinki Declaration. All participants signed written informed consent.

\section{Laboratory and ultrasonography measurements}

Anthropometric data, including age, gender, weight, and height, were recorded. Body mass index (BMI) was calculated as weight per square of height $\left(\mathrm{kg} / \mathrm{m}^{2}\right)$. Serum samples for laboratory analyses were obtained after overnight fasting. Total cholesterol, triglyceride (TG), high-density lipoprotein cholesterol (HDL-C), low-density lipoprotein cholesterol (LDL-C), glucose, glutamic-oxaloacetic transaminase (AST), glutamic-pyruvic transaminase (ALT), and homocysteine level were measured using a Dade Behring Dimension RXL Chemistry analyzer (Dade Behring Diagnostic, Marburg, Germany). We defined serum homocysteine of $15 \mu \mathrm{mol} / \mathrm{L}$ or greater as hyperhomocysteinemia and divided the participants into two groups (homocysteine $\geq 15 \mu \mathrm{mol} / \mathrm{L}$ and homocysteine $<15 \mu \mathrm{mol} / \mathrm{L}$ ) for further analysis.

The diagnosis of metabolic syndrome in our study was according to the 2004 Chinese Diabetes Society (CDS) criteria, ${ }^{18}$ at least three of the following components: (1) overweight or obesity (defined as BMI $\geq 25$ ); (2) fasting glycemia $\geq 6.1 \mathrm{mmol} / \mathrm{L}$ or previously diagnosed with type 2 diabetes, described as hyperglycemia; (3) blood pressure $\geq 140 / 90 \mathrm{mmHg}$ or previously diagnosed with hypertension, described as hypertension; or (4) TG concentration $\geq 1.7 \mathrm{mmol} / \mathrm{L}$ or previous TG abnormalities, described as hypertriglyceridemia, or HDL-C concentrations $<0.9 \mathrm{mmol} / \mathrm{L}$ in men and $1.0 \mathrm{mmol} / \mathrm{L}$ in women, described as low HDL-C.

All participants underwent a thyroid ultrasound test. We used Philips Healthcare iU22 xMATRIX sonographic instrument with $8 \mathrm{MHz}$ linear probes. Ultrasound examination was performed by the same radiologist who was blinded to clinical data. Thyroid nodule was defined as a lesion within the thyroid gland that has echoic distinction from the surrounding thyroid parenchyma.

\section{Statistical analyses}

The continuous data with normal distribution were expressed as mean \pm standard deviation. Non-normal distributed variables were presented as the medians, upper and lower quartiles. Categorical variables were expressed as numbers and percentages. Independent $t$-test or MannWhitney test was used for analyzing the continuous data between groups. Differences in categorical variables between groups were assessed with the chi-square test. Logistic regression analysis was used to identify whether the parameters were independent risk factors for the prevalence of thyroid nodule. Statistical analysis was performed using SPSS 21.0.

\section{Results \\ Prevalence of hyperhomocysteinemia and thyroid nodule}

Hyperhomocysteinemia were detected in 452 participants $(21.91 \%)$. Thyroid nodule prevalence was significantly higher in hyperhomocysteinemia participants than in normal homocysteine participants $(52.57 \%$ vs. $45.16 \%, P=0.006)$. Clinical parameters of hyperhomocysteinemia and normal homocysteine groups are shown in Table 1.

Table 1. Clinical Characteristics of the Participants with Hyperhomocysteinemia AND NoRmal HomOcysteine

\begin{tabular}{lccr}
\hline Variables & Homocysteine $\geq 15 \mu$ mol/L $(\mathrm{n}=449)$ & Homocysteine $<15 \mu \mathrm{mol} / \mathrm{L}(\mathrm{n}=1591)$ & $\mathrm{P}$ \\
\hline Age, years & $45.22 \pm 13.02$ & $43.72 \pm 11.54$ & 0.027 \\
Gender & $434(96.02)$ & $1256(77.92)$ & $<0.001$ \\
$\quad$ Male, $n(\%)$ & $18(3.98)$ & $356(22.08)$ & \\
$\quad$ Female, $n(\%)$ & $61(13.5)$ & $143(8.88)$ & 0.004 \\
Antihypertensive therapy, $n(\%)$ & $24(5.38)$ & $66(4.12)$ & 0.063 \\
Hypoglycemic therapy, $n(\%)$ & $25.44 \pm 3.41$ & $25.07 \pm 3.40$ & 0.045 \\
BMI, $\mathrm{kg} / \mathrm{m}^{2}$ & $131.26 \pm 20.05$ & $127.62 \pm 16.98$ & 0.001 \\
Systolic blood pressure, $\mathrm{mmHg}$ & $80.11 \pm 13.36$ & $77.67 \pm 11.78$ & 0.001 \\
Diastolic blood pressure, $\mathrm{mmHg}$ & $5.88 \pm 1.63$ & $5.71 \pm 1.05$ & 0.033 \\
Fasting glucose, mmol/L & $2.93 \pm 0.74$ & $2.86 \pm 0.53$ & 0.115 \\
LDL-C, mmol/L & $1.17 \pm 0.26$ & $1.25 \pm 0.31$ & $<0.001$ \\
HDL-C, mmol/L & $1.44(0.95-2.23)$ & $1.35(0.88-2.05)$ & 0.044 \\
TG, mmol/L & $23.58 \pm 11.52$ & $22.09 \pm 9.08$ & 0.011 \\
AST, U/L & $31.31 \pm 25.33$ & $28.85 \pm 20.51$ & 0.033 \\
ALT, U/L & $24.84 \pm 11.74$ & $11.19 \pm 9.85$ & $<0.001$ \\
Homocysteine, $\mu$ mol/L & $236(52.57)$ & $719(45.16)$ & 0.006 \\
Thyroid nodule, $n(\%)$ & & & \\
\hline
\end{tabular}

Data are presented as the mean $\pm \mathrm{SD}$, median (upper and lower quartiles), or $n(\%)$.

ALT, glutamic-pyruvic transaminase; AST, glutamic-oxaloacetic transaminase; BMI, body mass index; HDL-C, high-density lipoprotein cholesterol; LDL-C, low-density lipoprotein cholesterol; SD, standard deviation; TG, triglyceride;. 
Table 2. Clinical Characteristics of the Participants With and Without Thyroid Nodule

\begin{tabular}{|c|c|c|c|}
\hline Variables & Nodular group $(\mathrm{n}=955)$ & Non-nodular group $(\mathrm{n}=1085)$ & $\mathrm{P}$ \\
\hline Age, years & $47.42 \pm 11.59$ & $40.83 \pm 11.14$ & $<0.001$ \\
\hline Gender & & & 0.001 \\
\hline Male, $n(\%)$ & $756(79.16)$ & $918(84.61)$ & \\
\hline Female, $n(\%)$ & $199(20.84)$ & $167(15.84)$ & \\
\hline Antihypertensive therapy, $n(\%)$ & $132(13.82)$ & $69(6.37)$ & $<0.001$ \\
\hline Hypoglycemic therapy, $n(\%)$ & $41(4.37)$ & $34(3.14)$ & 0.145 \\
\hline $\mathrm{BMI}, \mathrm{kg} / \mathrm{m}^{2}$ & $25.42 \pm 3.449$ & $24.94 \pm 3.35$ & 0.002 \\
\hline Systolic blood pressure, $\mathrm{mmHg}$ & $130.26 \pm 19.011$ & $126.58 \pm 16.17$ & $<0.001$ \\
\hline Diastolic blood pressure, $\mathrm{mmHg}$ & $79.17 \pm 12.61$ & $77.24 \pm 11.74$ & 0.005 \\
\hline Fasting glucose, $\mathrm{mmol} / \mathrm{L}$ & $5.94 \pm 1.57$ & $5.75 \pm 1.42$ & 0.007 \\
\hline $\mathrm{LDL}-\mathrm{C}, \mathrm{mmol} / \mathrm{L}$ & $2.90 \pm 0.743$ & $2.86 \pm 0.758$ & 0.120 \\
\hline HDL-C, mmol/L & $1.23 \pm 0.307$ & $1.23 \pm 0.300$ & 0.341 \\
\hline $\mathrm{TG}, \mathrm{mmol} / \mathrm{L}$ & $1.38(0.90-2.08)$ & $1.36(0.89-2.11)$ & 0.819 \\
\hline $\mathrm{AST}, \mathrm{U} / \mathrm{L}$ & $22.60 \pm 10.27$ & $22.22 \pm 9.15$ & 0.375 \\
\hline ALT, U/L & $29.28 \pm 23.33$ & $29.46 \pm 20.212$ & 0.855 \\
\hline Homocysteine, $\mu \mathrm{mol} / \mathrm{L}$ & $14.57 \pm 8.887$ & $14.46 \pm 7.243$ & 0.027 \\
\hline Hyperhomocysteinemia, $n(\%)$ & $236(24.63)$ & $213(19.54)$ & 0.006 \\
\hline Metabolic syndrome, $n(\%)$ & $193(20.56)$ & $181(17.06)$ & 0.045 \\
\hline $\mathrm{BMI} \geq 25, n(\%)$ & $496(51.93)$ & $538(49.59)$ & 0.309 \\
\hline Hypertension, $n(\%)$ & $293(31.2)$ & $229(21.81)$ & $<0.001$ \\
\hline Hyperglycemia, $n(\%)$ & $256(26.95)$ & $213(19.69)$ & $<0.001$ \\
\hline Hypertriglyceridemia or low HDL-C, $n(\%)$ & $362(38.03)$ & $405(37.43)$ & 0.783 \\
\hline
\end{tabular}

Data are presented as the mean $\pm \mathrm{SD}$, median (upper and lower quartiles), or $n(\%)$.

\section{Comparison of clinical parameters and serum homocysteine level between thyroid nodule and non-thyroid nodule groups}

Characteristics of the participants with and without thyroid nodule are presented in Table 2. Of the 2040 adults, 955 $(46.81 \%)$ had thyroid nodule. Compared with subjects without nodule, patients with thyroid nodule were older, more likely to be female (all $P<0.05$ ). BMI, systolic blood pressure, diastolic blood pressure, fasting serum glucose, and homocysteine levels were significantly higher in patients with thyroid nodule than in those without thyroid nodule (all $P<0.05$ ). There were more patients who received antihypertensive therapy in nodular group than in non-nodular group (all $P<0.001$ ). The percentages of metabolic syndrome, hypertension, and hyperglycemia were significantly higher in nodular group than in non-nodular group (all $P<0.05$ ). There were no significant differences in TG, HDL-C, LDL-C, AST, and ALT between groups.

Table 3. Logistic Regression Analysis FOR THE ASSESSMENT OF INDEPENDENT RISK FACTORS of Prevalence of Thyroid Nodule

\begin{tabular}{|c|c|c|c|c|c|}
\hline Variables & $\beta$ & $S E$ & $O R$ & $95 \% C I$ & $\mathrm{P}$ \\
\hline ge & 0.053 & 0.005 & 1.055 & $1.045-1.064$ & $<0.001$ \\
\hline Gender & 0.878 & 0.142 & 2.406 & $1.820-3.179$ & $<0.001$ \\
\hline BMI & 0.049 & 0.016 & 1.050 & $1.018-1.083$ & 0.002 \\
\hline $\begin{array}{l}\text { Systolic blood } \\
\text { pressure }\end{array}$ & 0.006 & 0.005 & 1.006 & 0.996-1.016 & 0.192 \\
\hline $\begin{array}{l}\text { Diastolic blood } \\
\text { pressure }\end{array}$ & 0.005 & 0.007 & 1.003 & 0.989-1.015 & 456 \\
\hline Fasting glucose & 0.028 & 0.035 & 1.010 & 0.9 & 0.495 \\
\hline Homocysteine & 0.021 & 0.006 & 1.022 & $1.009-1.034$ & 0.001 \\
\hline
\end{tabular}

$\beta$, logistic regression coefficient; $\mathrm{CI}$, confidence interval; $\mathrm{OR}$, odds ratio; SE, standard error.

\section{Logistic regression analysis showing factors associated with thyroid nodule}

Multivariable logistic regression analysis revealed that age [odds ratio (OR) 1.054; 95\% confidence interval (CI) 1.046-1.062; $P<0.001]$, female gender (OR 2.242; 95\% CI 1.975-2.509; $P<0.001$ ), BMI (OR 1.050; 95\% CI 1.018$1.083 ; P<0.001$ ), and serum homocysteine level (OR 1.022; 95\% CI 1.009-1.034; $P=0.001)$ were the independent risk factors for thyroid nodule (Table 3 ).

\section{Discussion}

Our study showed that participants with hyperhomocysteinemia have significantly higher thyroid nodule prevalence. Serum homocysteine level is significantly higher in thyroid nodular group than in non-nodular group. Age, female gender, BMI, and serum homocysteine level were independent risk factors for thyroid nodule.

Thyroid nodule is the most common disorder of thyroid. Many studies have investigated the related risk factors of thyroid nodule. Our results indicate that advanced age, female gender, and BMI are independently associated with the prevalence of thyroid nodule, which is consistent with previous findings. ${ }^{3,7}$ It has been shown that there are estrogen receptors in thyroid follicular cells and $17 \beta$-estradiol could stimulate the growth of thyroid cells. ${ }^{19,20}$ This suggests that the gender difference might be due to the effect of estrogen. Some studies demonstrated that metabolic syndrome and its related components, including insulin resistance, obesity, increased blood glucose, dyslipidemia, and hypertension, contribute to the increased thyroid nodule prevalence. ${ }^{2,8,21}$

In this study, BMI, the systolic and diastolic blood pressure, and fasting serum glucose level were significantly higher in participants with thyroid nodule than in those without thyroid nodule. The percentages of metabolic 
syndrome, hypertension, and hyperglycemia were significantly higher in nodular group than in non-nodular group. However, the associations between blood pressure, fasting serum glucose level, and thyroid nodule disappeared after adjustment. In this study, more patients received antihypertensive therapy in thyroid nodular group than in non-nodular group. This probably decreased the association between blood pressure and thyroid nodule prevalence.

In our study, participants with hyperhomocysteinemia have significantly higher thyroid nodule prevalence than those with normal homocysteine. We also demonstrated that serum homocysteine level was an independent risk factor for thyroid nodule. The mechanism by which elevated homocysteine enhances thyroid nodule prevalence was not clear. Homocysteine is a sulfur-containing amino acid, whose metabolism is closely linked to cellular methylation status. There are two pathways of homocysteine metabolism, transsulfurating to cysteine and remethylating back to methionine. Methionine could be used for the synthesis of $S$-adenosylmethionine, a universal methyl donor for various methylation reactions, including the methylation of DNA, RNA, and protein. $S$-adenosylhomocysteine $(\mathrm{SAH})$ is a by-product of this methylation reaction, which can be hydrolyzed to homocysteine subsequently. This reaction is reversible with equilibrium dynamics. ${ }^{22}$ Elevated homocysteine concentration was found to be associated with increased concentrations of SAH. ${ }^{23}$ All the above indicates that the elevated homocysteine levels may have an influence on the cellular methylation stations, which were found to regulate cell proliferation, apoptosis, and differentiation in eukaryotes. $^{24,25}$ In addition, homocysteine was found to induce inflammation. ${ }^{13}$ Moreover, increased homocysteine was associated with several common adult cancers. ${ }^{14-17,26}$ Thus, elevated homocysteine may affect the growth and proliferation of thyroid cells, and promote nodular formation. Further animal studies are needed to clarify the mechanisms mediating the relationship between hyperhomocysteinemia and thyroid nodules.

Homocysteine concentration is regulated by both environmental and genetic factors. The intakes of folic acid and vitamin B are main environmental factors. The genetic factors include polymorphisms of the genes involved in homocysteine metabolism, such as methylenetetrahydrofolate reductase (MTHFR) 1298A $\rightarrow \mathrm{C}$ and $677 \mathrm{C} \rightarrow \mathrm{T} .{ }^{27}$ In this study, the average concentration of homocysteine of the whole population is $14.17 \mu \mathrm{mol} / \mathrm{L}$ and the prevalence of hyperhomocysteinemia (homocysteine $\geq 15 \mu \mathrm{mol} / \mathrm{L}$ ) is $22.0 \%$. This concentration of homocysteine is higher than some results reported in southern China and other countries. ${ }^{28-31}$ The population in this study was drawn from Beijing, which is in northern China, an area with low supplementation of folic acid and a high MTHFR 677C $\rightarrow$ T genotype frequencies. ${ }^{32,33}$ Further research in other areas would be necessary to test whether our results could be applied to other populations.

There are several limitations in this study. First, the sample size is not large. A larger sample size could lead to more definite conclusions. Second, because of the crosssectional design of this study, the temporal and causal association cannot be assessed. Third, the smoking history and the intakes of iodine, folic acid, or vitamin B were not recorded, and thus could not be included in the analyses. Many previous studies have demonstrated that smoking habit and iodine intake affect thyroid nodule prevalence. ${ }^{3}$
Therefore, further studies about the relationship of homocysteine and thyroid nodule are warranted.

\section{Conclusions}

Subjects with hyperhomocysteinemia have significantly higher thyroid nodule prevalence. Homocysteine is an independent risk factor for thyroid nodule. Our data indicate a possible pivotal role of homocysteine in thyroid nodule formation.

\section{Author Disclosure Statement}

No conflicting financial interests exist.

\section{Funding Information}

This study was supported by a grant from Beijing Hospitals Authority Youth Programme (code: QML20160304) to Q.P.

\section{References}

1. Pellegriti G, Frasca F, Regalbuto C, et al. Worldwide increasing incidence of thyroid cancer: Update on epidemiology and risk factors. J Cancer Epidemiol 2013;2013: 965212.

2. $\mathrm{Xu} \mathrm{W}, \mathrm{Chen} \mathrm{Z}, \mathrm{Li}$ N, et al. Relationship of anthropometric measurements to thyroid nodules in a Chinese population. BMJ Open 2015;5:e008452.

3. Jiang H, Tian Y, Yan W, et al. The prevalence of thyroid nodules and an analysis of related lifestyle factors in Beijing Communities. Int J Environ Res Public Health 2016;13:442.

4. Chen Z, Xu W, Huang Y, et al. Associations of noniodized salt and thyroid nodule among the Chinese population: A large cross-sectional study. Am J Clin Nutr 2013;98:684692.

5. Tae HJ, Lim DJ, Baek KH, et al. Diagnostic value of ultrasonography to distinguish between benign and malignant lesions in the management of thyroid nodules. Thyroid 2007; 17:461-466.

6. Guth S, Theune U, Aberle J, et al. Very high prevalence of thyroid nodules detected by high frequency $(13 \mathrm{MHz}) \mathrm{ul}-$ trasound examination. Eur J Clin Invest 2009;39:699-706.

7. Panagiotou G, Komninou D, Anagnostis P, et al. Association between lifestyle and anthropometric parameters and thyroid nodule features. Endocrine 2017;56:560-567.

8. Ayturk S, Gursoy A, Kut A, et al. Metabolic syndrome and its components are associated with increased thyroid volume and nodule prevalence in a mild-to-moderate iodinedeficient area. Eur J Endocrinol 2009;161:599-605.

9. Moon JH, Hyun MK, Lee JY, et al. Prevalence of thyroid nodules and their associated clinical parameters: A largescale, multicenter-based health checkup study. Korean $J$ Intern Med 2018;33:753-762.

10. Guba SC, Fink LM, Fonseca V. Hyperhomocysteinemia. An emerging and important risk factor for thromboembolic and cardiovascular disease. Am J Clin Pathol 1996;106: 709-722.

11. Sreckovic B, Sreckovic VD, Soldatovic I, et al. Homocysteine is a marker for metabolic syndrome and atherosclerosis. Diabetes Metab Syndr 2017;11:179-182.

12. Wang Y, Jiang Y, Wang N, et al. Central but not general obesity is positively associated with the risk of hyperhomocysteinemia in middle-aged women. Nutrients 2019;11:1614. 
13. Elsherbiny NM, Sharma I, Kira D, et al. Homocysteine induces inflammation in retina and brain. Biomolecules 2020;10:393.

14. Wu LL, Wu JT. Hyperhomocysteinemia is a risk factor for cancer and a new potential tumor marker. Clin Chim Acta 2002;322:21-28.

15. Zhang D, Wen X, Wu W, et al. Elevated homocysteine level and folate deficiency associated with increased overall risk of carcinogenesis: Meta-analysis of 83 case-control studies involving 35,758 individuals. PLoS One 2015; 10:e0123423.

16. Keshteli AH, Baracos VE, Madsen KL. Hyperhomocysteinemia as a potential contributor of colorectal cancer development in inflammatory bowel diseases: A review. World J Gastroenterol 2015;21:1081-1090.

17. Hasan T, Arora R, Bansal AK, et al. Disturbed homocysteine metabolism is associated with cancer. Exp Mol Med 2019;51:21.

18. Wen J, Yang J, Shi Y, et al. Comparisons of different metabolic syndrome definitions and associations with coronary heart disease, stroke, and peripheral arterial disease in a rural Chinese population. PLoS One 2015;10:e126832.

19. Furlanetto TW, Nguyen LQ, Jameson JL. Estradiol increases proliferation and down-regulates the sodium/iodide symporter gene in FRTL-5 cells. Endocrinology 1999;140: 5705-5711.

20. Manole D, Schildknecht B, Gosnell B, et al. Estrogen promotes growth of human thyroid tumor cells by different molecular mechanisms. J Clin Endocrinol Metab 2001;86: 1072-1077.

21. Shin J, Kim MH, Yoon KH, et al. Relationship between metabolic syndrome and thyroid nodules in healthy Koreans. Korean J Intern Med 2016;31:98-105.

22. Finkelstein JD. Pathways and regulation of homocysteine metabolism in mammals. Semin Thromb Hemost 2000;26: 219-225.

23. James SJ, Melnyk S, Pogribna M, et al. Elevation in S-adenosylhomocysteine and DNA hypomethylation: Potential epigenetic mechanism for homocysteine-related pathology. J Nutr 2002;132:2361S-2366S.

24. Pan Y, Liu G, Zhou F, et al. DNA methylation profiles in cancer diagnosis and therapeutics. Clin Exp Med 2018;18: $1-14$.

25. Stephen JK, Chen KM, Merritt J, et al. Methylation markers differentiate thyroid cancer from benign nodules. J Endocrinol Invest 2018;41:163-170.
26. $\mathrm{Xu} J$, Zhao $X$, Sun $\mathrm{S}$, et al. Homocysteine and digestive tract cancer risk: A dose-response meta-analysis. J Oncol 2018;2018:3720684

27. Clarke R, Bennett DA, Parish S, et al. Homocysteine and coronary heart disease: Meta-analysis of MTHFR casecontrol studies, avoiding publication bias. PLoS Med 2012; 9:e1001177.

28. Ganji V, Kafai MR. Population reference values for plasma total homocysteine concentrations in US adults after the fortification of cereals with folic acid. Am J Clin Nutr 2006; 84:989-994.

29. Wang Y, Li X, Qin X, et al. Prevalence of hyperhomocysteinaemia and its major determinants in rural Chinese hypertensive patients aged 45-75 years. Br J Nutr 2013; 109:1284-1293.

30. Chen KJ, Pan WH, Yang FL, et al. Association of B vitamins status and homocysteine levels in elderly Taiwanese. Asia Pac J Clin Nutr 2005;14:250-255.

31. Jacques PF, Rosenberg IH, Rogers G, et al. Serum total homocysteine concentrations in adolescent and adult Americans: Results from the third National Health and Nutrition Examination Survey. Am J Clin Nutr 1999;69: 482-489.

32. Huo Y, Li J, Qin X, et al. Efficacy of folic acid therapy in primary prevention of stroke among adults with hypertension in China: The CSPPT randomized clinical trial. JAMA 2015;313:1325-1335.

33. Yang B, Liu Y, Li Y, et al. Geographical distribution of MTHFR C677T, A1298C and MTRR A66G gene polymorphisms in China: Findings from 15357 adults of Han nationality. PLoS One 2013;8:e57917.

Address correspondence to: Guang Wang, MD

Department of Endocrinology Beijing Chao-Yang Hospital Capital Medical University No. 8, Gongti South Road, Chaoyang District Beijing 100020

China

E-mail:drwg6688@163.com 